\title{
THE AFFINE STRUCTURES ON THE REAL TWO-TORUS. I
}

\author{
BY T. NAGANO ${ }^{1}$ AND K. YAGI
}

Communicated by S. S. Chern, April 17, 1973

We wish to complete the study of the affine structures on the real affine 2-tori $T^{2}$, following N. H. Kuiper [2], J. P. Benzecri [1] and others. The category of the affine manifolds is defined, as usual, by the manifolds equipped with maximal atlas whose coordinate transformations are affine transformations $y^{i}=\Sigma_{j} a_{j}^{i} x^{j}+b^{i}, a_{j}^{i}, b^{i} \in R$, in the cartesian space $R^{n}$, and by the maps which are expressed locally with affine transformations in terms of the affine charts.

Our main result asserts that the affine structures on $T^{2}$ are completely determined by the holonomy groups, in which, however, the concept of the holonomy group requires a slight modification as follows.

Given an affine manifold $M$, its universal covering manifold $M^{\sim}$ with the induced affine structure is immersed equidimensionally into $R^{n}$ by an affine map $d$. The map $d$ gives rise to a homomorphism $\eta: \pi_{1}(M) \rightarrow$ $A\left(R^{n}\right)$ of the fundamental group into the affine group $A\left(R^{n}\right)$ in such a way that $d$ is $\pi_{1}(M)$-equivariant with respect to the action of $\pi_{1}(M)$ on $R^{n}$ through $\eta$. The image of $\eta$ is called the holonomy group $H$ of $M$, which is unique up to an inner automorphism of $A\left(R^{n}\right)$. Here $A(M)$, in general, denotes the affine automorphism group of the affine manifold $M$. When the image $d M^{\sim}$ is not simply connected, we switch to its universal covering $\left(d M^{\sim}\right)^{\sim}$ from $R^{n}$; that is, we construct an affine immersion: $d^{*}: M^{\sim} \rightarrow\left(d M^{\sim}\right)^{\sim}$ which covers $d$ and a homomorphism $\eta^{*}: \pi_{1} M \rightarrow$ $A\left(\left(d M^{\sim}\right)^{\sim}\right)$ accordingly. Now the modified holonomy group $H^{*}$ of $M$ is by definition the image $\eta^{*}\left(\pi_{1} M\right)$. When $d M^{\sim}$ is simply connected, we simply put $H^{*}=H$. At any rate $H^{*}$ can be regarded as a subgroup of the universal covering group $A\left(R^{2}\right)^{\sim}$ of $A\left(R^{2}\right)$.

THEOREM 1. Two affine structures on $T^{2}$ are isomorphic if and only if the modified holonomy groups are conjugate in $A\left(R^{2}\right)^{\sim}$.

The difficulty in the proof lies in establishing that $d$ is a covering map onto $d M^{\sim}$. The difficulty may be illustrated by the fact that a surjective immersion of $R^{2}$ onto itself is not always a diffeomorphism. In any case, that $d$ is a covering implies that $T^{2}$ is affine isomorphic with $\left(d M^{\sim}\right)^{\sim} / H^{*}$. In order to describe the classification of $H^{*}$ it is convenient to state the following theorem.

AMS(MOS) subject classifications (1970). Primary 53C05, 57D15.

${ }^{1}$ Partially supported by NSF GP-29662. 
THEOREM 2. For any affine torus $T^{2}$, the affine group $A\left(T^{2}\right)$ admits nonempty open orbits.

In the transitive case, $H^{*}$ is characterized as a lattice subgroup $\cong Z^{2}$ of a maximal connected abelian subgroup $G^{*} \cong R^{2}$ of $A\left(R^{2}\right)^{\sim}$. The projection $G=\pi\left(G^{*}\right)$ of $G^{*}$ in $A\left(R^{2}\right)$ is listed below. Since $G^{*}$ acts on the affine plane $R^{2}$ almost effectively, $G^{*}$ has the induced affine structure, and so $G^{*} / H^{*}$ becomes an affine torus naturally. In the intransitive case, the situation is more complicated; the affine 2-torus $T^{2}$ is then partitioned into several, say $n$, isomorphic open cylinders and their boundaries (which are closed geodesics in one and the same homotopy class $\alpha$ in $\pi_{1}\left(T^{2}\right)$; those cylinders together constitute the open orbit of $\mathrm{A}\left(T^{2}\right)$ ). To be more precise, $T^{2}$ has a cylinder $R \times S^{1}$ as an affine (regular) covering space which admits the affine transformations $\beta(k):(x, y) \rightarrow$ $(x+k, y), k \in Z$, and the covering group is generated by $\beta(n) . H^{*}$ is contained in a 2-dimensional abelian subgroup $G^{*}$ of $A\left(R^{2}\right)^{\sim}$ which is saturated (viz. $G^{*}=\pi^{-1} \pi\left(G^{*}\right)$ ) with respect to the projection $\pi: A\left(R^{2}\right)^{\sim} \rightarrow$ $A\left(R^{2}\right)$ and whose image under $\pi$ has the identity component $G$ of type (I-1) or (I-2) in the list below. In particular $\pi\left(G^{*}\right)$ is a linear transformation group having no translation part. $\pi(G)$ is generated by $G$ and the reflection, -1 , with respect to the fixed point of $G . G^{*}$ is isomorphic with $\operatorname{Ker} \pi \times \pi\left(G^{*}\right) \cong Z \times G$. Now $H^{*}$ is generated by two members $\alpha^{*}, \beta^{*}$ such that we have $\alpha^{*}=(0, \alpha)$ and $\beta^{*}=\beta(n)=(n, \beta)$ in the above correspondence, and that $\alpha$ is expanding (viz. the eigenvalues of the linear map $\alpha$ are greater than one and this is a characterization of $H^{*}$ ).

A question yet to be answered would be: What is the whole picture of all the affine structure of $T^{2}$ ? We intend to answer this question in a forthcoming paper.

Finally we list the conjugate classes of the maximal abelian connected subgroups $G$ of $A\left(R^{2}\right)$, writing $\left(\begin{array}{lll}a & b & p \\ c & d & q\end{array}\right)$ for the affine transformation $(x, y) \rightarrow(a x+b y+p, c x+d y+q)$. $G$ consists of
$(\mathrm{I}-1):\left(\begin{array}{ccc}a & b & 0 \\ 0 & a & 0\end{array}\right)$
(III-1): $\left(\begin{array}{lll}1 & b & p \\ 0 & 1 & b\end{array}\right)$,
$(\mathrm{I}-2):\left(\begin{array}{lll}a & 0 & 0 \\ 0 & d & 0\end{array}\right)$,
(III-2): $\left(\begin{array}{lll}1 & 0 & p \\ 0 & 1 & q\end{array}\right)$,
$(\mathrm{I}-3):\left(\begin{array}{rrr}u & v & 0 \\ -v & u & 0\end{array}\right)$,
(III-3): $\left(\begin{array}{lll}1 & b & p \\ 0 & 1 & 0\end{array}\right)$,
(II): $\left(\begin{array}{lll}1 & 0 & p \\ 0 & d & 0\end{array}\right)$, 
1973] THE AFFINE STRUCTURES ON THE REAL TWO-TORUS. I 1253

where $a>0, d>0,(u, v) \neq(0,0)$ and the others are arbitrary real numbers.

\section{REFERENCES}

1. J. P. Benzecri, Variétés localement affines, Séminaire Ehresmann, May 1959.

2. N. H. Kuiper, Sur les surfaces localement affines. Géométrie différentielle, Colloq. Internat. Centre National de la Recherche Scientifique, Strasbourg; Centre National de la Recherche Scientifique, Paris, 1953, pp. 79-87. MR 15, 648.

Department of Mathematics, University of Notre Dame, Notre Dame, Indiana 46556

Department of Mathematics, Osaka University, OSAKa, Japan 